Revista de Antropología Social

ISSN: 1131-558X

https://dx.doi.org/10.5209/raso.65613

\title{
(Des)encuentros entre las instituciones y la economía social y solidaria en Cataluña ${ }^{1}$
}

\author{
Patricia Homs Ramírez de la Piscina ${ }^{2}$
}

Recibido: 10 de diciembre de 2018/ Aceptado: 28 de marzo de 2019

Resumen. Desde 2008 las políticas de ajuste estructural han desregulado el mercado laboral en España. Después de la destrucción de miles de puestos de trabajo a lo largo de estos últimos años, se ha señalado al sector de la economía social y solidaria, y en particular a las cooperativas de trabajo, como empresas "resilientes" durante los periodos de crisis. Algunas instituciones han tomado medidas para promover este sector de "más rápida recuperación" haciendo inversiones directas en los proyectos de economía social y solidaria, proporcionando facilidades burocráticas y financieras y ofreciendo asesoramiento y formación gratuita en esta área. El artículo aborda el papel de las instituciones en el presente crecimiento de la economía social y solidaria en Cataluña y los efectos de esta estrecha relación a través de diversos casos etnográficos. ¿Se están socializando las prácticas o se están vaciando de su contenido político? ¿Quién está estableciendo la agenda de esta articulación? ¿Cuáles son las mayores tensiones que emergen en estas relaciones? ¿Cuáles son los efectos de este crecimiento para las sociastrabajadoras de las cooperativas?

Palabras clave: Cooperativas; crisis; resiliencia; instituciones; economía social y solidaria.

\section{[en] (Mis)matches between institutions and social and solidarity economy in Catalonia}

\begin{abstract}
Since 2008, structural adjustment policies have deregulated the labor market in Spain. After the destruction of thousands of jobs over the last years, the social and solidarity economy and, in particular, worker cooperatives have been pointed out as "resilient" enterprises during periods of crisis. Some institutions have taken measures to promote this "faster recovery" sector by making direct investments in social and solidarity economy projects, providing bureaucratic and financial facilities and offering advice and free training in this area. The article addresses the role of institutions in the current growth of the social and solidarity economy in Catalonia and the effects of this close relationship through various ethnographic cases. Are the practices being socialized or are they being emptied of their political content? Who is setting up the agenda for this articulation? What are the greatest tensions that emerge in these relationships? What are the effects of this growth for cooperative workers?
\end{abstract}

Keywords: Cooperatives; crisis; resilience; institutions; social and solidarity economy.

Sumario. 1. La crisis y el desempleo como motores de la auto-ocupación. 2. Casos etnográficos. 3. Reconfiguraciones de las formas de trabajo: lo político y lo económico. 4. La resiliencia como resistencia. 5. El papel de las instituciones en el desarrollo de la Economía Social y Solidaria (ESS). 6. La Economía Social y Solidaria en los intersticios del mercado capitalista. 7. A modo de conclusión. 8. Bibliografía.

1 La autora quiere agradecer el soporte del proyecto "Grassroots Economics: Meaning, Project and Practice in the Pursuit of Livelihood" [GRECO], PI Narotzky, European Research Council Advanced Grant 2012, IDEASERC FP7, Project Number: 323743, 2013-2018, durante la presente investigación.

2 GRECO, Universitat de Barcelona; L’Aresta Cooperativa. patihoms@gmail.com 
Cómo citar: Homs Ramírez de la Piscina, P. (2019). (Des)encuentros entre las instituciones y la economía social y solidaria en Cataluña, en Revista de Antropología Social 28(2), 227-246.

\section{La crisis y el desempleo como motores de la auto-ocupación}

Desde la crisis de los setenta, el desempleo ha sido uno de los pilares que ha justificado y validado las reformas en el sistema productivo y las medidas políticas que han precarizado la vida de miles de personas (Seminario de Economía Crítica Taifa, 2006). En este sentido, el desempleo es un elemento productivo que ha legitimado las intervenciones políticas y ha reasignado a los individuos la responsabilidad de tener o no trabajo a través de los discursos de la empleabilidad y el emprendimiento.

Asimismo, a lo largo de estas últimas cinco décadas, la legislación laboral española se ha centrado, siguiendo las recomendaciones europeas, en bajar los salarios, reducir los costes de contratación y despido, y socavar los derechos sociales adquiridos tras las luchas obreras. Como consecuencia, las negociaciones colectivas se han reconfigurado en negociaciones individuales para cada contrato con el objetivo de asegurar las necesidades de acumulación de capital de las empresas. La protección laboral ha menguado, hasta el punto que el obrero es señalado como responsable de no encontrar trabajo, ya sea por su cualificación insuficiente o por carecer de espíritu emprendedor. De modo que se han impulsado la auto-ocupación y el emprendimiento como ejes fundamentales en diversas áreas y a distintas escalas: en la educación primaria y secundaria, en los programas de inserción laboral, en la formación para desempleados, en la formación profesional para trabajadores en activo, etc. (Martínez y Bogino, 2015).

En este sentido, las cooperativas son proyectos de auto-ocupación colectiva que incorporan valores añadidos en relación a los proyectos individuales de emprendimiento, como son la cooperación, la solidaridad, la resiliencia, la economía social, etc. Estos valores refuerzan el discurso moral, los beneficios sociales, la vinculación comunitaria, e incluso evocan empoderamiento y transformación social. Así, las cooperativas y la Economía Social y Solidaria (ESS) encajan y están respaldadas por el contexto actual de retraimiento en materia de bienestar por parte del Estado. Efectivamente, los recortes sociales y la supuesta devolución del poder a los individuos, a las comunidades y a la sociedad civil, juntamente con el desarrollo del ethos emprendedor, están en el corazón de la Big Society (Jacobs y Manzi, 2013; McCabe, 2010; Oppenheim y Platt, 2010).

Los datos de los diferentes sectores laborales en España y Cataluña a lo largo de esta última crisis socioeconómica y financiera indican que las cooperativas fueron las primeras en experimentar un cambio positivo en relación al resto de sectores: la destrucción de lugares de trabajo acabó en 2011 y se empezó a generar empleo en 2013. Ese mismo año también se empezaron a crear lugares de trabajo en el área de los trabajadores autónomos dados de alta en el Régimen Especial de Trabajadores Autónomos (RETA); mientras que el trabajo asalariado no empezó a presentar cifras positivas hasta el 2014. Esta evolución es idéntica al caso catalán, salvo que en esta comunidad se adelantó un año la creación de empleo en todos los sectores. 
Figura 1. Evolución del mercado laboral en España y en Cataluña.

Tasas de variación anual de 2008 a 2016.
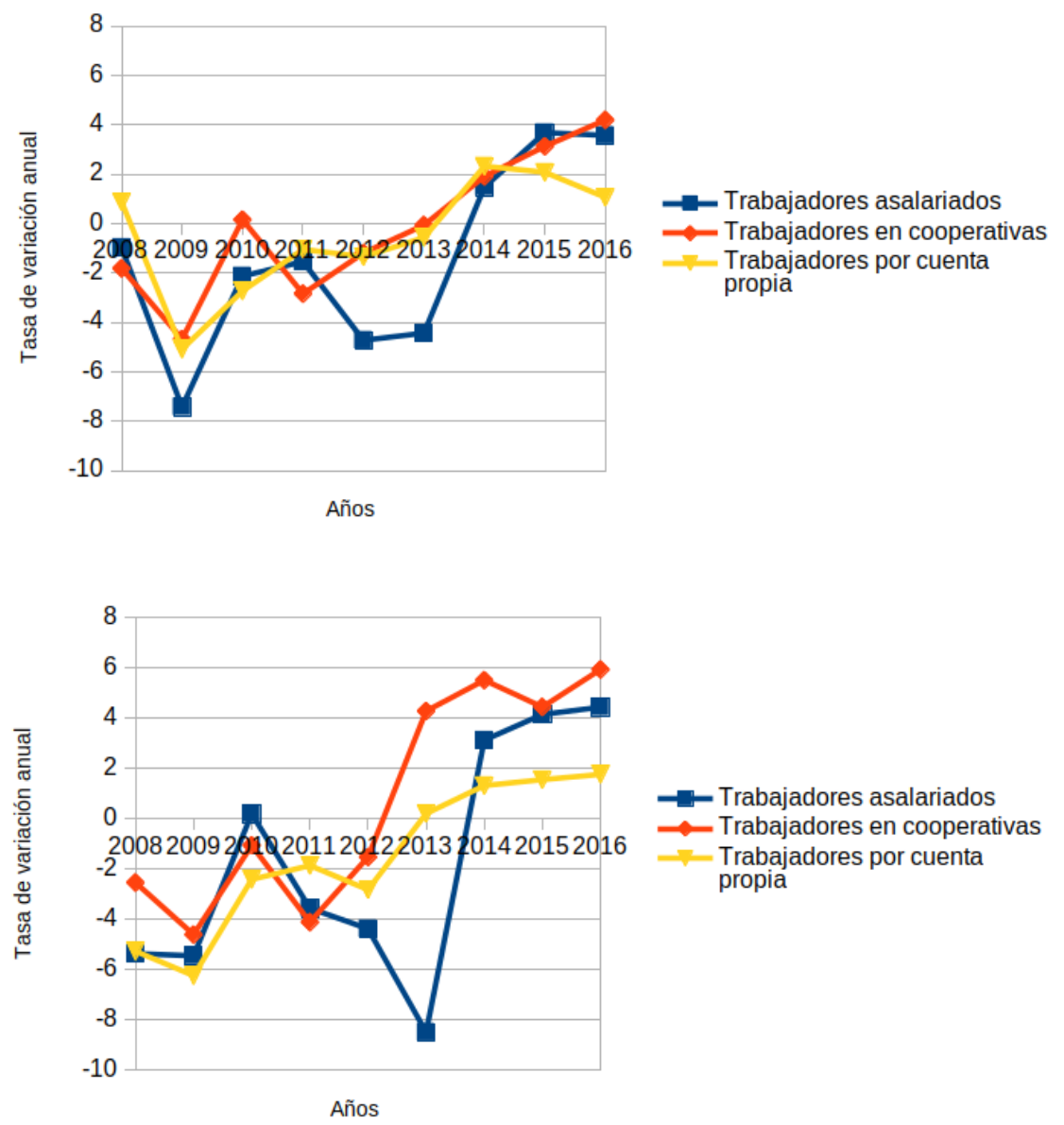

Fuente: Elaboración propia a partir de datos del Ministerio de Empleo y Seguridad Social.

Sin embargo, el número de cooperativas, tanto en España como en Cataluña, disminuyó entre los años 2008 y 2012. En el 2013 hubo una ligera recuperación, pero en el 2014 sufrieron de nuevo un descenso del que empezaron a recuperarse al año siguiente. 
Figura 2. Evolución del número de cooperativas en España y Cataluña. Tasas de variación anual de 2008 a 2016 y número de cooperativas en el periodo 2007-2016.

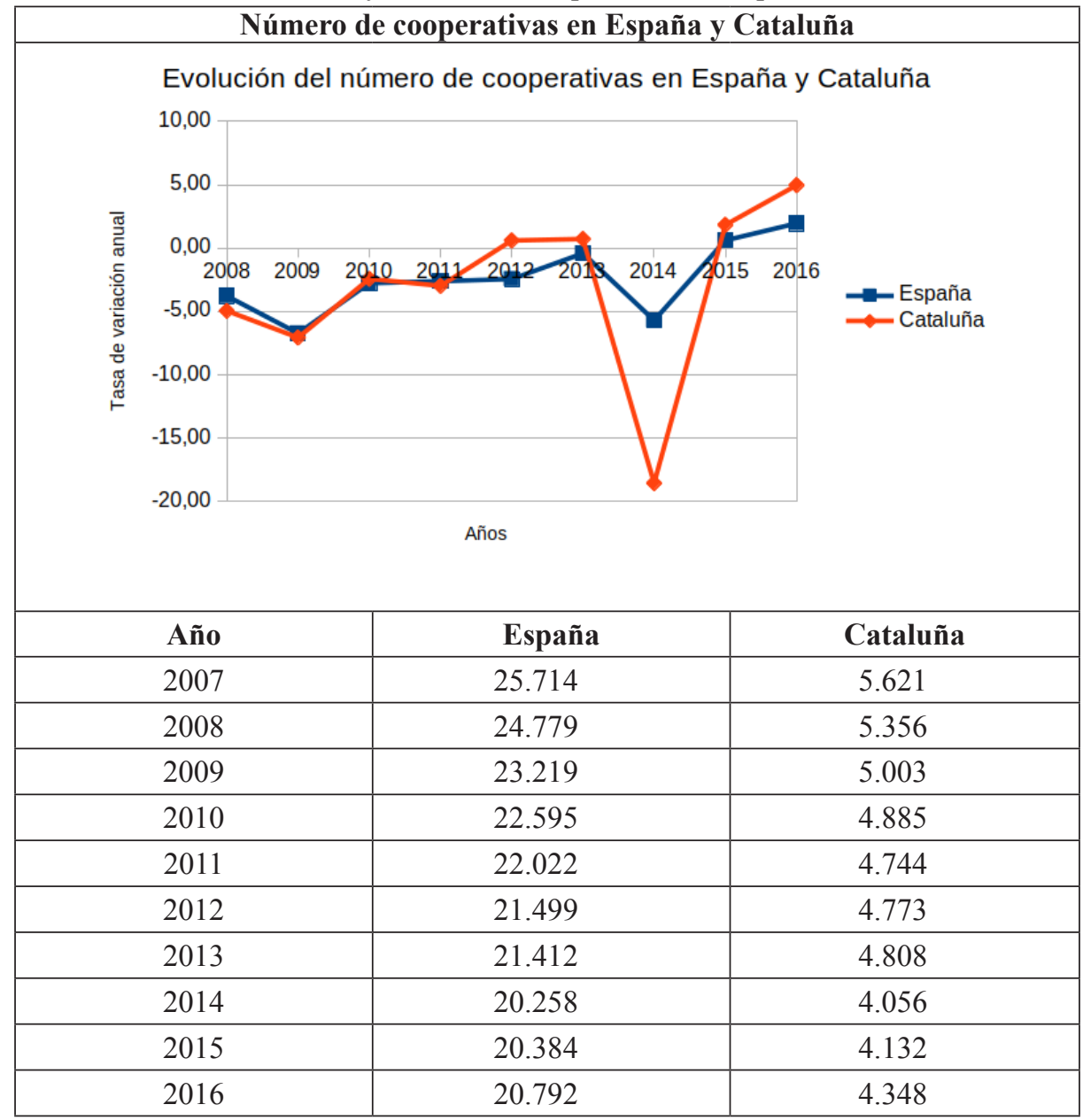

Fuente: Elaboración propia basada en datos del Ministerio de Empleo y Seguridad Social.

Por consiguiente, si combinamos los datos del número de cooperativas y del número de trabajadores en este sector en Cataluña, vemos cómo el número de cooperativas presenta tasas de crecimiento más bajas que el número de trabajadores, indicando que el sector ha tendido a la concentración, es decir, menos cooperativas con más trabajadores (Fernández y Miró, 2016). De tal modo que se han seguido estrategias similares a las de las empresas capitalistas para reducir los costes e incrementar la acumulación de capital durante el último periodo de austeridad.

Asimismo, no hay estudios minuciosos que detallen y valoren la calidad de los puestos de trabajo: ¿los trabajadores son también socios de la cooperativa? ¿están registrados en el RETA o en el régimen general? ¿cuál es la duración del contrato? Consideramos que estos datos son esenciales para poder analizar la resiliencia de las cooperativas (Kasmir, 2016). En la investigación hemos podido acceder a algunos datos que revelan que desde 2014 ha habido un incremento importante en el número 
de cooperativas afiliadas al RETA ${ }^{3}$ en Cataluña (Castell, Ferre, Olivella et al., 2016). El porcentaje de cooperativas afiliadas a este régimen fue de 58,8\% en $2014,63 \%$ en 2015 y $67 \%$ en 2016 . Esta inclinación se ha manifestado desde 1999, cuando solo el $41 \%$ de las cooperativas estaban afiliadas al RETA. Siguiendo esta misma tendencia, el porcentaje de trabajadores autónomos en las cooperativas ha aumentado desde el $27 \%$ en 1999 hasta el $36 \%$ en 2016. Estas cifras se alinean con la tendencia al autoempleo que en España se traduce en ser trabajador autónomo y, en consecuencia, estar dado de alto en el RETA 4 .

No obstante, aparte de los discursos sobre la resiliencia y los beneficios sociales de las cooperativas que han promovido el crecimiento de la ESS gracias al apoyo institucional, también tenemos que considerar otros factores que explican la relevancia y el crecimiento de las cooperativas y, en general, de la ESS en el territorio catalán. Por un lado, la larga tradición cooperativista de la región desde que a mediados del siglo XIX se crearan las primeras cooperativas; esta tradición ha continuado hasta el presente con sus altos y bajos (Pérez Baró, 1989). Por otro lado, el movimiento 15M que desborda las plazas de España y Cataluña en 2011. El 15M cuestiona el sistema socioeconómico capitalista y apunta a las cooperativas como iniciativas socioeconómicas centradas en las personas y no en el capital como posibles alternativas a la economía hegemónica. Tras el 15M hay un aumento muy significativo en el número de socios de cooperativas financieras como Coop57 o de consumo como Som Energia. Además, la Red de Economía Solidaria (Xarxa d'Economia Solidària, XES) organizó la primera feria catalana de la economía solidaria (Fira d'Economia Solidària de Catalunya, FESC) en octubre de 2012. Este evento significó la primera puerta de entrada a la ESS para mucha gente. Asimismo, la XES desde el 2015 ha promovido la ESS en las economías municipales a través de la estrategia de las "14 medidas para impulsar la ESS a nivel local" y la formación de núcleos locales de ESS. Finalmente, también hay que destacar algunos cambios políticos en la administración que han facilitado la expansión y crecimiento del cooperativismo y la ESS, especialmente en el crecimiento de las cooperativas de trabajo. Primero, los cambios en los gobiernos municipales como el del Ayuntamiento de Barcelona desde la victoria de Barcelona en Comú en mayo de 2015. Segundo, el cambio en la Dirección General de Economía Social, Cooperativismo, Tercer sector y Autoempresa del Gobierno catalán a principios del 2016.

A pesar de estos factores más regionales, diversos autores han mostrado cómo el soporte institucional hacia estas formas socioeconómicas tiende a incrementarse durante periodos de crisis en parte para asegurar la paz social (Bouchard, Bourque y Lévesque, 2000; Lautier, 2003; Salvia, 2004; Moulaert y Ailenei, 2005). De tal modo que, en periodos de disconformidad social, la energía social debe redirigirse para asegurar la continuidad del sistema de mercado capitalista. En este sentido, se

Las cooperativas de trabajo pueden estar dadas de alta en el régimen general o en el RETA. Cada cooperativa puede escoger el régimen, pero es obligatorio que todos los socios trabajadores estén dados de alta en el mismo régimen. Una vez la cooperativa ha escogido uno de los regímenes, los miembros solo pueden cambiar su afiliación al cabo de cinco años.

4 En este artículo no abordamos el conflicto de los falsos autónomos; trabajadores que realizan los trabajos para una empresa pero que no son trabajadores por cuenta ajena -a pesar de que cumplen todos los requisitos- sino que se les obliga a estar dados de alta en el RETA para que la empresa ahorre la cotización a la Seguridad Social. Además, también hay que tener en cuenta que hay trabajadores que deberían estar dados de alta en el RETA, pero para ahorrarse los costes de las cuotas mensuales no están dados de alta de forma permanente sino solo en los meses de facturación. 
han realizado diversas recomendaciones a nivel europeo para reorientar las energías sociales productivas hacia el mercado (Salvatori, 2011; COM, 2011; EGSEC, 2016). Además, la promoción de cooperativas durante los periodos de crisis y de escasez de trabajo asalariado también se puede interpretar como un programa específico de emprendimiento colectivo en un marco general hegemónico de promoción de la auto-ocupación -véanse los programas específicos en emprendimiento de la Comisión Europea de los años 1998 (Promoting entrepreneurship in Europe: priorities for the future), 2004 (The European program for entrepreneurship) y 2013 (2020 Entrepreneurship Action Plan).

Finalmente, la resiliencia es uno de los factores que legitima la asignación de recursos a las cooperativas y ha facilitado la expansión del cooperativismo y la ESS. En este artículo deconstruimos el uso hegemónico del término y lo resignificamos desde una perspectiva doble. Por un lado, como la capacidad de adaptación a las demandas de capital a través de la intensificación y extensión de la precarización y explotación capitalista en los proyectos de auto-ocupación colectiva. Por otro lado, como una práctica de resistencia cotidiana que se encarna en los cuerpos de los y las cooperativistas. Del mismo modo, observamos cómo estos procesos tensionan el conflicto capital/vida hasta el punto que los trabajadores y las trabajadoras ven amenazada la sostenibilidad de sus vidas a lo largo del desarrollo de los proyectos de ESS (Pérez-Orozco, 2012; 2014; Federici, 2013).

\section{Casos etnográficos}

El trabajo de campo de esta investigación incluye 10 meses de observación participante en el área de Cataluña a lo largo de los años 2017 y 2018, y 19 entrevistas semi-estructuradas a informantes clave del movimiento cooperativista teniendo en cuenta, entre otros factores, la antigüedad de los proyectos, el sector de trabajo de la cooperativa y el vínculo con la administración pública. También se ha llevado a cabo un estudio estadístico sobre el mercado laboral y un análisis de diversos documentos y políticas públicas a diferentes escalas espaciales: europea, nacional, regional y programas locales. Además, se han analizado los resultados de diez cuestionarios a diez socios y socias de cooperativas de trabajo, centrados en temas relacionados con la salud, la resiliencia y el emprendimiento.

En este artículo se presentan principalmente los resultados de cuatro casos de estudio. Primero, dos ateneos cooperativos ${ }^{5}$; cada ateneo está formado por una red de diversas entidades, principalmente pertenecientes al sector de la economía social y solidaria. En uno de ellos también participa la administración pública a través de ayuntamientos, consejos comarcales y agentes de desarrollo local. Estas redes están completamente subvencionadas por el Gobierno catalán desde octubre del 2016 con el objetivo de promocionar la fórmula cooperativa. A lo largo del 2018 se ha debatido sobre la continuidad de estas redes como un servicio público-comunitariocooperativo. Los objetivos principales de los ateneos cooperativos son visibilizar e

\footnotetext{
Estos dos ateneos están liderados por cooperativas relativamente pequeñas (menos de 8 socios) con un posicionamiento político que persigue la transformación social. Ambas tratan de consolidar los puestos de trabajo y tienen la voluntad que todos los trabajadores y trabajadoras sean socios-trabajadores. Las personas que trabajan en ambas entidades tienen un largo recorrido en movimientos sociales y acciones socio-políticas.
} 
impulsar la creación de empresas cooperativas y mejorar el mercado laboral en las distintas áreas territoriales a través de las mismas. El tercer caso de estudio es un programa formativo de emprendimiento colectivo que se ha llevado a cabo de 2013 a 2016 en la ciudad de Barcelona. Este programa ofrecía herramientas prácticas, conocimiento y recursos a proyectos con "vocación de transformación social" diferencia de la ideología convencional del emprendedor individual que invisibiliza las desigualdades socioeconómicas estructurales, este curso formativo partía de las diferencias iniciales y no responsabilizaba al individuo de su éxito o fracaso. Finalmente, el cuarto caso de estudio es la XES, fundada formalmente en 2003, y que a lo largo de estos últimos 14 años ha experimentado un crecimiento importante hasta convertirse en uno de los mayores referentes de la ESS en Cataluña. En 2018, la XES estaba formada por más de 100 miembros, 15 entidades de soporte y 190 entidades con actividad económica.

El trabajo de campo también se ha llevado a cabo en instituciones vinculadas a estos cuatro casos de estudio (Ayuntamiento de Barcelona, especialmente el Comisionado de Economía Social y Solidaria; Barcelona Activa, el servicio de ocupación municipal; la Dirección General de Economía Social, el Tercer Sector, las Cooperativas y la Autoempresa de la Generalitat de Catalunya). Asimismo, se ha realizado trabajo de campo en otras entidades, cooperativas, asociaciones e instituciones de la región.

El artículo aborda el papel de las instituciones en el presente crecimiento de la economía social y solidaria en Cataluña y los efectos de esta estrecha relación a través de casos etnográficos. ¿Se están socializando las prácticas o se están vaciando de su contenido político? ¿Quién está estableciendo la agenda de esta articulación? ¿Cuáles son las mayores tensiones que emergen en estas relaciones? En el artículo también analizamos el discurso que relaciona el auge de las cooperativas y la ESS con los periodos de crisis capitalista y la vinculación con algunas de las supuestas particularidades de estas fórmulas empresariales como la resiliencia. A lo largo del trabajo de investigación nos hemos preguntado quién y cómo se sostiene la resiliencia de estas experiencias socioeconómicas.

\section{Reconfiguraciones de las formas de trabajo: lo político y lo económico}

En el discurso público de políticos y técnicos de la administración, la resiliencia aparece como un elemento clave para justificar la promoción de cooperativas en un contexto de crisis: se trata de fórmulas empresariales que soportan mejor los periodos difíciles. Sin embargo, los miembros de las cooperativas no se refieren a ella cuando explican sus motivaciones para involucrarse o crear una iniciativa empresarial cooperativa. La mayoría ${ }^{7}$ consideran que el proyecto de economía social y solidaria ha sido una manera de encontrar -o más bien, crear- un trabajo, y que la

\footnotetext{
A lo largo del articulo, las comillas indican que se trata de palabras literales de grabaciones o de material escrito. Todas las cooperativas que han participado en esta investigación son relativamente pequeñas (con un mínimo de tres socios y un máximo de ocho), están vinculadas a movimientos sociales y a la transformación social. Según algunos autores, este tipo de microcooperativas pertenecen al modelo solidario debido a sus valores en la organización interna. Este modelo se distinguiría de las cooperativas mercantilizadas en tanto que no se basan en la competitividad y la búsqueda de beneficios económicos (Mansilla, Grenzner y Alberich, 2014).
} 
fórmula cooperativa es la que mejor encajaba con sus prácticas y posicionamientos políticos. Así lo relata un socio de una cooperativa:

Teníamos muy claro que queríamos ser una cooperativa de trabajo. De hecho, cuando teníamos estas discusiones que te estaba comentando antes en [un colectivo de principios del 2000], pusimos sobre la mesa las alternativas que nos ofrecía el mercado laboral (...) La lógica de ser autónomos y todas estas cosas no nos atraía y la fórmula cooperativa era la más adecuada para dar continuidad a la asamblea. (Socio-trabajador de una cooperativa de Barcelona con 13 años de antigüedad y miembro de un ateneo cooperativo).

O tal como lo expresa un socio-trabajador de una cooperativa de vivienda de una zona rural fundada hace dos años y también miembro de un ateneo cooperativo:

Había unas redes de gente del mundo de las alternativas políticas, sociales y económicas y todo; bueno, la crisis los ha empujado un poco a desarrollar economía social y solidaria. Vamos a llevar a la práctica lo que siempre estamos diciendo en todos los sitios donde militamos, vamos a aplicarlo a nuestro trabajo cotidiano, a nuestra economía.

En estos casos, la crisis se interpreta como una oportunidad para reconfigurar la acción política de activistas sociales en actividades económicas. Tal como atestiguan las palabras de un cooperativista que elabora vino de nueces de forma artesanal, la resiliencia es "la pasión por el trabajo cuando uno emprende por militancia, cuando considera que la cooperativa es política". En efecto, algunos autores han declarado que la mayoría de las cooperativas pequeñas de la región catalana tienen sus raíces en los movimientos sociales del territorio (Mansilla, Grenzner y Alberich, 2014).

Ahora bien, esta articulación entre lo político y lo económico no está libre de tensiones (Martín-Mayor, Homs y Flores-Pons, 2017). Por un lado, hay activistas que taxativamente rechazan mezclar la esfera política y la económica, tal como queda reflejado en las siguientes palabras de una miembro de una cooperativa de consumo:

Yo siempre he creído que cobrar de tu militancia, o cobrar de lo que tu crees, que sea tu medio económico de vida, tu pasión, del tipo que sea, te mete en aguas pantanosas. Es muy complicado. Es un problema porque aquí se están haciendo las cosas desde la pasión desde una convicción política y así, pero además es tu medio de vida. (...) en cuanto estamos con los pies en el mercado, se te complica todo hasta unos términos, (...) hay que hilar muy fino, para no salirse y no liarla, y no hacer de tu discurso, tu necesidad, y de tu necesidad, el discurso, y... ser coherente, tanto consumidores como proveedores, la coherencia y el mantenerte, o el escepticismo: "pues mira, es que es mi trabajo, chicos, ahora no hablamos de política".

Por otro lado, tal como se observa en las dos primeras citas de este apartado, otros tratan de crear proyectos políticos con el objetivo de que sean su medio de vida y, en consecuencia, lidiar con los aspectos económicos. En este sentido, algunos proyectos explican sus objetivos como cooperativa en la línea de: "transferir el conocimiento de la auto-organización y la autogestión al área del trabajo y la economía" (extraído de la web: http://www.laciutatinvisible.coop/, un proyecto cooperativo de librería, 
asesoramiento y formación). También encontramos posiciones más extremas como la que argumenta un miembro de una cooperativa cultural: "Intuíamos que la lucha sociopolítica que no generara alternativas socioeconómicas no era transformadora ni era sostenible". Estas aproximaciones están de acuerdo con las economías feministas que expresan la necesidad de volver a poner la economía en manos de la política (Pérez-Orozco, 2012).

A lo largo del trabajo de campo hemos observado que las personas que acceden a auto-ocuparse a través de una cooperativa forman parte de una fracción de la clase trabajadora con cierto nivel de capital cultural, social y económico. Por un lado, se trata de activistas con estudios de grado superior y, generalmente, con una gran cantidad de contactos y miembros de diversas redes. Por otro lado, no tienen una necesidad económica urgente ya que logran mantenerse con salarios muy bajos, especialmente durante los primeros años de apertura de la empresa. Además, en general, son individuos con hábitos de consumo ascéticos (Bourdieu, 1988: 185) y sin otras personas a su cargo, cosa que facilita la sostenibilidad económica de sus vidas.

En este sentido, estamos ante un sector de clase social con ciertos privilegios en las dimensiones culturales, sociales y económicas. Sin embargo, la crisis ha abierto un desajuste entre las expectativas materiales y laborales y su realización. Así, la crisis, más allá de la ausencia de trabajo asalariado, es una crisis de reproducción social que empeora las condiciones de vida y genera una rotura de las expectativas de futuro (Narotzky y Besnier, 2014).

No obstante, vemos cómo las condiciones reales en las que se encuentran los y las cooperativistas, una vez iniciado el proyecto, también se mueven entre dos ejes. Primero, hay una precarización del trabajo en relación a las horas trabajadas y los sueldos cobrados. Precisamente la dimensión política configurada a menudo en términos morales es la que invisibiliza o justifica, durante al menos un tiempo, la precarización económica. En este contexto, el desajuste material en relación a las expectativas laborales no se reajusta al trabajar en una cooperativa, sino que en la mayoría de los casos se agrava. Segundo, la condición de socio-trabajador les sitúa en una nueva posición desde la cual pueden contratar a trabajadores y trabajadoras. Esto ubica a los socios-trabajadores en un lugar de cierto privilegio pero que queda socavado en parte cuando, en algunos casos, los trabajadores y trabajadoras tienen mejores condiciones laborales y menos responsabilidades que las socias. De nuevo, esta situación se invisibiliza o justifica con afirmaciones morales que realzan la dimensión política del proyecto: "pero es que en verdad es muy guay formar parte de Tasera ${ }^{8}$. Poder decir que eres socio de Tasera y tomar las decisiones de la cooperativa es guay, te hace estar contento y sentirte afortunado" (notas diario de campo).

Cerramos este apartado retomando la idea de que, en periodos de crisis, se intensifica el cuestionamiento del sistema socioeconómico y las cooperativas emergen como una posible vía para canalizar las motivaciones políticas en el seno de un proyecto económico. Esta reflexión contrasta con los análisis macroeconómicos que describen la trayectoria contra-cíclica de las cooperativas en relación a la expansión de capital. A saber, las cooperativas supondrían un refugio de empleo en momentos

Nombre ficticio de una cooperativa de trabajo dedicada a la producción alimentaria, en la que, tras cinco años de recorrido, las socias-trabajadoras dadas de alta en el régimen de autónomos, cotizando la mínima base salarial, siguen cobrando menos que las trabajadoras contratadas en régimen general. A lo largo de todo el artículo se han cambiado los nombres de las personas, así como los de las cooperativas. 
de crisis con tendencia a reconvertirse en empresas capitalistas en periodos de expansión (Ben-Ner, 1988). En estas interpretaciones, se confrontan índices como el PIB o la tasa de paro con el balance neto de cooperativas, mostrando una correlación directa entre ambos parámetros (Serrano, Crespo, Celma et al., 2017). En el caso de Cataluña, vemos cómo en la última crisis del 2008, a diferencia de las anteriores, no hay una correlación inmediata. De hecho, en el 2008 se destruyeron un gran número de cooperativas llegando a la desaparición del 20\% de las cooperativas en algunos sectores como el de la construcción. De tal modo que no es hasta el 2015, tras el inicio de un periodo de mayor apoyo institucional, que no se observa de nuevo una recuperación de las cooperativas de trabajo.

\section{La resiliencia como resistencia}

El concepto de resiliencia se desarrolló sobre los años 70 en las ciencias ecológicas para indicar la persistencia de las relaciones dentro de un sistema incluso tras el impacto de perturbaciones externas. De tal modo que la resiliencia se define como la capacidad para absorber cambios de estado provocados por fuerzas externas. El concepto se ha trasladado a las ciencias sociales y algunos autores proponen que el éxito del término se debe a su encaje con la ideología neoliberal de los sistemas complejos adaptativos (Walker y Cooper, 2011). El capitalismo necesita naturalizar espacios de resiliencia que atañan a las demandas cambiantes de acumulación de capital de una economía globalizada (MacKinnon y Driscoll, 2012). A pesar de que el término ha recibido diversas críticas (Evans, 2011), es ampliamente usado por expertos y por la administración pública así como por activistas sociales y miembros de movimientos anticapitalistas (Walker y Cooper, 2011; MacKinnon y Driscoll, 2012).

Desde 2008 las políticas de ajuste estructural han generado una desregulación del mercado laboral en España. Después de la destrucción de miles de puestos de trabajo a lo largo de estos últimos años, se ha señalado al sector de la economía social y solidaria y, en particular, las cooperativas de trabajo como empresas más "resilientes". Algunas instituciones han tomado medidas para promover este sector de "más rápida recuperación" haciendo inversiones directas en los proyectos de economía social y solidaria, proporcionando facilidades burocráticas y financieras, y ofreciendo asesoramiento y formación gratuita en esta área. Ahora bien, este discurso legitimador de las prácticas institucionales se sustenta sobre categorías propias de la ideología económica ortodoxa que separa lo público, lo productivo y lo mercantil de lo privado, improductivo y doméstico, y que explica la crisis por la reducción de trabajo asalariado y un aumento del desempleo. Ahora bien, hay otras aproximaciones a la economía que la redefinen en el ámbito de la reproducción social (Narotzky, 2004; Narotzky y Besnier, 2014) y que permiten explorar otros niveles de la crisis y, en este caso concreto, los de la resiliencia.

En un primer nivel, como ya hemos visto previamente, el incremento de cooperativas se da a partir del 2015 cuando el sector empieza a recibir apoyo institucional. No obstante, el mayor aumento son empresas cooperativas dadas de alta en el régimen de autónomos y faltan datos en relación a la supervivencia de las nuevas cooperativas que permitan afirmar que se están generando puestos de trabajo más estables $\mathrm{y}$ de calidad. Tal como nos comentaba un investigador y socio de una cooperativa cultural con 13 años de antigüedad: 
Las cooperativas incrementan el número de personas no a través de los socios sino a través de contratos de trabajo. Por lo tanto, hay una reducción de la democracia cooperativa durante los años de crisis. (...) Cuando se dice que la ocupación en el cooperativismo se ha mantenido durante la crisis, es verdad. Pero hay que mirar que tipo de ocupación y que tipo de relación societaria tiene esta ocupación.

Más allá del discurso macroeconómico público, los informantes detallan un segundo nivel en el que la resiliencia se explica porque, en las cooperativas, los trabajadores son los dueños de la empresa y siguen estrategias de reestructuración de costes y ajustamiento de salarios según la situación socioeconómica. En este sentido, podemos interpretar la resiliencia como una doble práctica de adaptación y resistencia con el objetivo de sostener el proyecto durante los periodos de crisis. Así, las cooperativas superan etapas difíciles gracias a su capacidad de internalizar las consecuencias de la crisis a través de los trabajadores de la empresa; en concreto, la estrategia adaptativa más efectiva y de uso habitual es la reducción de los salarios (Mansilla, Grenzner y Alberich, 2014). En palabras de un socio-trabajador de una cooperativa dedicada a la difusión cultural: "La resiliencia de las cooperativas, creo que está muy basada en las capacidades que tienen, ahora de mover jornadas, mover salarios...no ha sido un camino de rosas, muchas veces ha sido a expensas de los socios de la cooperativa (...) pero también es verdad que lo han hecho voluntariamente".

Este punto de vista es compartido por algunas personas que trabajan en las instituciones y que a la vez tienen vínculos con los movimientos sociales. Por ejemplo, uno de los técnicos del Ayuntamiento de Barcelona y activista de la XES afirmaba:

No nos vamos a engañar, la economía social y solidaria es muy militante, son proyectos que vienen desde abajo y esto llega a unos extremos, no quiero decir de autoexplotación pero...jornadas muy duras porque estás luchando por tu proyecto. Este es un valor muy importante, por eso, hay unos niveles de implicación muy altos, porque estás trabajando para tu proyecto. Todo esto hace que sea más resiliente.

De tal modo que la resiliencia es una manera de "asumir la precariedad de forma colectiva", una manera "de compartir las vulnerabilidades" o, tal como lo expresan varios informantes, "una autoexplotación colectiva". Las estrategias adoptadas en las cooperativas de trabajo tienen cierta similitud con las descritas en contextos rurales por autores como Friedman (1978), Chayanov (1991) y Galt (2013), como el retraso en el pago de salarios, la intensificación de la producción o el aumento de la jornada laboral. Además de esta resiliencia estratégica y adaptativa, observamos un tercer nivel de resiliencia encarnada en los cuerpos de los trabajadores y trabajadoras. Todos los informantes que trabajan en las cooperativas han relatado una serie de malestares concerniendo a su salud y su cuerpo:

Acabo agotado, muy agotado del cerebro, muy agotado, muy agotado, durante los fines de semana, intento desconectar; agotado de la cantidad de cosas que tengo en la cabeza (...) yo me agoto mentalmente, por la noche, o el viernes, ya no puedo 
más. No me caben más cosas en el cerebro, pero físicamente...no hago deporte, fumo, estoy gordo. De repente un día entraré en urgencias.

Los y las cooperativistas nos han explicado un sinfín de dolores físicos y psicológicos que relacionan con su trabajo ${ }^{9}$, como son episodios de estrés y ansiedad, tensiones, insomnio, agotamiento, cansancio, dolor de espalda, dolores musculares, dolores de barriga, dolores de cabeza, migrañas, dolor cervical y lumbar y, en general, tener las defensas bajas, cosa que aumenta las probabilidades de enfermar. Estos resultados están en consonancia con estudios anteriores que muestran cómo los trabajadores y trabajadoras de las cooperativas están exhaustos físicamente y emocionalmente estresados (Kasmir, 2016). Este agotamiento físico y mental se expresa en una compresión de los espacios de vida y trabajo (Sarkis, 2018) en los que las personas sobrecargadas no pueden "parar de hacer, pero con la sensación que no llegas a todo" (notas diario de campo), y no pueden separar dentro de su actividad cooperativizada el continuo entre activismo, trabajo y vida.

La resiliencia de las cooperativas se encarna en los cuerpos de los trabajadores y trabajadoras ${ }^{10}$, una resiliencia íntima que se sostiene sobre las vidas que asumen la responsabilidad y el compromiso afectivo y moral con el proyecto; creando islas de sobrecarga de trabajo en un paisaje de falta de trabajo asalariado. La resiliencia sería a la vez el reflejo público de la corporalización de las consecuencias del contexto socioeconómico, así como una resistencia a este mundo capitalista que trata de subyugar cada una de las esferas de la vida (Narotzky, 2004; Federici, 2013; Alquézar, Homs, Morelló et al., 2014). De tal modo que las diferentes escalas de la resiliencia conectan la dimensión macroeconómica (y macropolítica) con la microeconómica (y micropolítica), rompiendo las dicotomías entre el mercado y lo doméstico, lo productivo y lo improductivo, lo público y lo privado (Elson, 2001).

\section{El papel de las instituciones en el desarrollo de la Economía Social y Solidaria (ESS)}

Algunos autores destacan que mientras durante otras crisis el cooperativismo ha resistido y reaccionado antes que otros sectores en materia de empleo, después de la crisis del 2008 no se ha producido tal efecto hasta que el movimiento ha recibido el apoyo institucional a partir del 2015. En este sentido, parte de las entidades de la ESS han iniciado o estrechado relaciones ya existentes con las instituciones a lo largo de estos últimos cuatro años. En el transcurso de esta colaboración ha tenido lugar una gran variedad de situaciones y experiencias ${ }^{11}$.

Primero, los ritmos de trabajo de los proyectos que generalmente implican procesos de auto-organización son más lentos que los que exigen las instituciones. En este sentido, diversos miembros se lamentan de que la relación con las insti-

\footnotetext{
9 Los socios-trabajadores que llevan menos de tres años en la cooperativa consideran que estos malestares son comunes en todos los trabajos o al menos son propios de todos los proyectos de emprendimiento. Por lo tanto, no constituirían una particularidad de las cooperativas.

10 Consideramos de gran interés profundizar en la diversidad de cuerpos según el género, la edad, etc. y ver como la crisis de reproducción social los afecta de forma distinta.

11 En este apartado recogemos aquellas situaciones y relatos que consideramos cualitativamente o cuantitativamente más relevantes.
} 
tuciones haya acelerado y, por tanto, alterado procesos que hubiesen seguido otra velocidad y recorrido. Un caso concreto, y que se ha repetido en diferentes contextos, es que en muchas de las convocatorias para optar a subvenciones se valora el hecho de que los y las solicitantes estén agrupados en una red de cooperativas o de entidades vinculadas a la ESS. Ahora bien, en muchos casos esas redes no existían previamente a la convocatoria y se han creado rápidamente para poder presentarse a estas. En consecuencia, se parte de unas redes que no se han creado tras procesos de trabajo cooperativo previo, sino que se construyen a posteriori en caso de salir beneficiados de las ayudas institucionales. Otro ejemplo que ilustra con claridad esta aceleración de ritmos de trabajo es el de una cooperativa que se constituyó a la luz de la convocatoria de los ateneos cooperativos y que se lamenta que se han volcado en este proyecto, olvidando su actividad como cooperativa de vivienda a la que un socio afirma que dedica entre cinco y diez horas semanales: "Fue un proceso muy acelerado [la creación de la cooperativa]. Hay que hacerlo ya porque si hacemos esto del ateneo... fue muy en paralelo, o sea que acabó siendo muy instrumental, la Tamonoco; porque el ateneo ha hecho casi que... dedicamos muy pocas horas a lo que pensábamos que queríamos dedicar."

Segundo, a lo largo del establecimiento de los acuerdos con la administración se han generado cambios importantes en las líneas estratégicas y objetivos de las entidades de la ESS. Es lo que un informante miembro de la XES nos detallaba como "hipertrofia de las líneas estratégicas", "la hoja de ruta desdibujada" o "el norte se redibuja" tras empezar a trabajar con la administración local. Así, el plan de trabajo viene condicionado por quien otorga los recursos y no existe una creación compartida de abajo a arriba. Del mismo modo, en muchos casos los indicadores de evaluación son propuestos íntegramente por la administración, limitando las posibilidades de desarrollo de los proyectos.

Tercero, algunas entidades han desagregado sus identidades en tanto que la identidad de la cooperativa se separa de la identidad de participación en el proyecto en el que se colabora con la institución. Este es el caso de una cooperativa que participa del grupo motor de un ateneo cooperativo y ha decidido en la medida de lo posible no vincular su identidad a la participación en este proyecto subvencionado por la $\mathrm{Ge}-$ neralitat de Cataluña. Además, dos socios explicaron en una asamblea cómo desde el ateneo cooperativo se mantienen posicionamientos y discursos, con la pretensión de llegar a un público más amplio, que no se podrían asumir fácilmente desde la cooperativa porque implicaría un debate muy largo y posiblemente un desacuerdo en algunos aspectos.

Cuarto, los cambios que se han producido en los últimos años en las instituciones, concretamente en el Ayuntamiento de Barcelona, en Barcelona Activa y en la Dirección General de Economía Social han permitido que algunos activistas ocupen "posiciones de más poder y más estratégicas" dentro de las instituciones. Ante esta coyuntura, algunas voces se lamentan de que a lo largo de estos años se haya producido una descapitalización y desmovilización de los movimientos sociales. De todos modos, hay que tener en cuenta que la propia participación de activistas en las cooperativas también puede entenderse como una desmovilización ya que reiteradamente, en las entrevistas y en la práctica, se observa un desplazamiento de toda su actividad política a la actividad de la cooperativa. Siguiendo la línea anterior, algunas personas que participan de la ESS consideran que las instituciones se exceden en la cantidad de actos oficiales a los que se les invita a participar, incluso en algún caso 
como representación institucional. Sin embargo, algunos informantes han relatado que el hecho que las instituciones estén tan cercanas a los movimientos sociales permite que "podamos usar las instituciones para objetivos colectivos".

Quinto, a lo largo del trabajo de campo también hemos observado que la administración se ha acercado a algunos colectivos de la economía informal y ha elaborado estudios jurídicos para evaluar su posible formalización. Este ha sido el caso del Ayuntamiento de Barcelona y las denominadas economías comunitarias en las que se incluyen los grupos de consumo, los huertos urbanos, los grupos de crianza o las redes de intercambio. En este sentido, se propuso la creación de un espacio de participación de las economías comunitarias con el objetivo de detectar las necesidades del sector y ver cómo el Ayuntamiento podía incidir en su satisfacción. Uno de los temas que ha ido surgiendo a lo largo de estos encuentros es la elaboración de estudios jurídicos para evaluar las diferentes posibilidades de formalización de los grupos de crianza y los grupos de consumo.

Finalmente, según algunos expertos, la interacción entre la ESS y la economía mercantil también ha generado la exportación de valores propios de las cooperativas a las empresas capitalistas, como la participación, la intercooperación ${ }^{12}$ y el interés comunitario. Por otro lado, la ESS y en concreto las cooperativas han importado valores como la efectividad y el marketing de las empresas mercantiles (Mateu, 2016). En esta misma línea, muchos informantes realzan la importancia de los "procesos de hibridación" o "contaminación" entre las cooperativas y la economía mercantil. Tal como explica el activista y cargo político en el Ayuntamiento de Barcelona:

Y esto significa salir de los espacios de confort y conectar con gente que no viene de un proceso político tan maduro (...) creo que es bueno contaminarnos de otros sectores más convencionales porque así podremos mirar algunas cosas con menos complejidad: meritocracia, marketing, rentabilidad, crédito...

En esta misma línea, desde el programa de emprendimiento colectivo que ha participado en esta investigación se interpreta este tipo de emprendimiento como una oportunidad concreta para hibridar el conocimiento de la gestión colectiva, procedente de los movimientos sociales, y el conocimiento de la viabilidad económica procedente del área empresarial. Esta aproximación es vista como una oportunidad que facilitará tanto la sostenibilidad económica como la sociabilización de la ESS.

No obstante, la expansión de la ESS presenta ciertas dificultades en la medida que algunos cooperativistas detallan que no hay suficiente presión social y que se sigue estando en una situación de "más oferta que demanda". En efecto, varios cooperativistas consideran que el gran motor de cambio se encuentra en el ámbito del consumo. Sin embargo, el acceso a bienes y servicios procedentes de la ESS es limitado, especialmente debido a la dificultad de acceso y los precios más elevados. En este sentido, vemos como la administración local impulsa la ESS desde diferentes perspectivas en función de las realidades socioeconómicas. Así, el Ayuntamiento de

12 En este artículo no desarrollamos el papel que juega la intercooperación y la creación de mercado social en el crecimiento de la ESS. Ahora bien, consideramos que se trata de estrategias con un papel importante en el desarrollo de la ESS, y que sería conveniente continuar profundizando en ellas. 
Barcelona ha promocionado el consumo de bienes y servicios procedentes de la ESS en los cuatro barrios con más poder adquisitivo de la ciudad. En contraposición, se han invertido más recursos en la creación de puestos de trabajo en el área de la ESS en aquellos barrios con menor poder adquisitivo y con más problemáticas asociadas al desempleo. Ahora bien, desde el Ayuntamiento se reconoce la dificultad de alcanzar este objetivo porque, como hemos detallado anteriormente, crear un proyecto de auto-ocupación colectiva implica no encontrarse en una situación de necesidad económica urgente ${ }^{13}$. En esta línea, algunos activistas se preguntan si las instituciones que están promocionando este tipo de iniciativas de fomento de la ESS están efectivamente participando en los circuitos de economía reales. Es decir, se están financiando proyectos de ESS pero ¿realmente la administración pública se aprovisiona con los bienes y servicios de estos circuitos? ${ }^{14}$

Los casos de estudio de esta etnografía muestran procesos bottom-up a lo largo de su creación y desarrollo, pero la situación actual de la ESS supone que su sostenibilidad y crecimiento en parte se mantiene por la financiación del Estado y la privada. Esta dependencia genera que su expansión y líneas estratégicas estén a menudo condicionadas por aquellos que financian los proyectos. Asimismo, crecer dependiendo de las subvenciones no asegura un crecimiento estable y consolidado, $\mathrm{y}$, muy a menudo, está vinculado a la contratación de trabajadores asalariados temporales sin la posibilidad efectiva de que se incorporen como socios y socias de la cooperativa ${ }^{15}$. Algunos cooperativistas plantean, desde la informalidad, que este vínculo está creando una "burbuja de la economía social y solidaria". Sin embargo, en espacios más formalizados se ha abierto un debate sobre este término. En estos espacios aparecen posiciones argumentadas para oponerse a dicha terminología porque genera una "vinculación con el sector especulativo", transmite una idea de "fragilidad, vacío y vulnerabilidad", en cualquier momento puede "pincharse y desaparecer", etc. En este sentido, se prefiere centrar la cuestión en abrir el debate sobre el crecimiento de la ESS y el papel de las instituciones en dicho crecimiento, así como sus riesgos y oportunidades. Además, hablar de "burbuja" invisibiliza que la "administración aún dedica pocos recursos a la ESS" y "sigue financiando mayoritariamente la economía capitalista". Asimismo, difumina que existe un "interés social propio previo al soporte institucional". En el debate titulado "La burbuja de la ESS", que tuvo lugar en la FESC del 2018, se apuntó que tal vez sí que se puede hablar de burbuja en tanto que a lo largo del crecimiento se están reproduciendo las mismas limitaciones que las de la economía convencional. Es decir, la ESS está volcada en el sector servicios, pero sigue habiendo muy pocos proyectos relacionados con el sector primario y el industrial. Por otra parte, a pesar de los riesgos y dependencias que implica el sopor-

13 Diversos estudios realizados por el IGOP (Institut de Govern i Polítiques Públiques) muestran como la innovación social se da en lugares sin segregación socioecómica. En efecto, no encontramos innovación en aquellos barrios desfavorecidos con pocos recursos ni tampoco en aquellos con mayor poder adquisitivo. Este contexto tiende a favorecer la segmentación socioeconómica y a aumentar las desigualdades -véanse los resultados del proyecto «Barris i Crisi»: https://barrisicrisi.wordpress.com/

14 En el transcurso de esta investigación se ha publicado una nueva guía de contratación pública del Ayuntamiento de Barcelona que incluye ciertas claúsuslas sociales. La XES ha acompañado este proceso cediendo el conocimiento adquirido a través de herramientas propias como el Balance social.

15 Hay que considerar que algunos de los servicios de acompañamiento a la creación y consolidación de cooperativas difícilmente podrán sostenerse sin las subvenciones directas. Sin embargo, algunas cooperativas y, en general, los ateneos cooperativos se están planteando como mejorar su sostenibilidad a través de la diversificación de fuentes de financiamiento, la generación de actividad económica propia y la búsqueda de fondos europeos. 
te institucional, los informantes valoran que sin este soporte muchos de los proyectos estarían en una situación de mayor precariedad.

\section{La Economía Social y Solidaria en los intersticios del mercado capitalista}

Las iniciativas socioeconómicas enmarcadas en la ESS están guiadas por la lógica de la vida, la reproducción ampliada de la vida (Coraggio, 2004), y no por la lógica de la acumulación capitalista. Ahora bien, la producción de bienes y servicios inevitablemente pasa a través del mercado capitalista.

A menudo estas experiencias son encajadas en el marco teórico de las economías diversas (Gibson-Graham, 2008) o de la economía plural (Laville, 2013). En general, este tipo de propuestas teóricas invisibilizan la resistencia necesaria para la supervivencia de estas iniciativas socioeconómicas en un mundo capitalista. Además, la coexistencia supuestamente armoniosa de diversas formas económicas no muestra las tensiones y luchas, sino que presupone un paisaje diverso y optimista en el que las empresas sociales irán lentamente reemplazando a las empresas capitalistas (Gibson-Graham, 2006). Esta reflexión de corte más teórico se ejemplifica en nuestro caso de estudio a través de la resiliencia de las cooperativas. El discurso público invisibiliza las tensiones y luchas materiales, concretas y reales que encarnan los cuerpos de los cooperativistas que tratan de resistir en el día a día tirando hacia delante un proyecto que aúna lo político y lo socioeconómico, pero también la vida.

Estas iniciativas quieren ser sostenibles en un sentido amplio del término: social, política, ambiental y económicamente; y situar la vida en el centro de todo proceso económico (Federici, 2013; Pérez-Orozco, 2014). No obstante, en el contexto actual deben operar en el mercado y, finalmente, los principios y valores mercantiles acaban estructurando su organización y parte de sus prácticas (Homs y Narotzky, 2019). Así, aunque haya una serie de criterios políticos que definan y delimiten estos proyectos, la esfera del intercambio sigue guiada por los valores de competencia y efectividad capitalistas (Álquezar, 2015; Kasmir, 2016).

El discurso público de la resiliencia se apoya en el relato hegemónico de la crisis basado en la falta de empleo, e invisibiliza la crisis de reproducción social que genera un exceso de trabajo tanto remunerado como no, una intensificación y extensión de las relaciones capitalistas de explotación y la transferencia de valores añadidos a los proyectos. En relación a este último punto, hemos recogido a lo largo del trabajo de campo una serie de valores que oscilan desde poner al servicio de la cooperativa las redes de confianza y reciprocidad de los miembros hasta valores basados en el esfuerzo personal al describirse el trabajo "como una actividad pasional que va más allá del trabajo", "que tiene que ver con la acción política", una manera de luchar por la justicia social o incluso como una "enfermedad" propia de los cooperativistas que acabaría justificando el poner el proyecto socioeconómico en el centro de la vida.

En el contexto actual, las instituciones juegan un papel esencial en el desarrollo de la ESS a través de su financiamiento, redefinición de objetivos y líneas estratégicas y marcando los ritmos de trabajo. Tal como he recogido en otro artículo (Homs, 2019), la articulación entre el emprendimiento y el cooperativismo es un caso claro de encuentro entre dinámicas top-down originadas en las instituciones y bottom-up de las economías populares, que ha suscitado diversos posicionamientos en el seno de la ESS. Aparte de los pocos proyectos que se han posicionado políticamente en 
contra del uso del discurso del emprendimiento, hay una idea generalizada de que dicha articulación configura una oportunidad para la ESS. En primer lugar, se mantiene una visión utilitarista cuando se defiende que, gracias al uso de los canales del emprendimiento convencional, se puede llegar a más gente. En segundo lugar, la imbricación ha permitido acceder a recursos económicos, lo que de otro modo no hubiese sido posible. Finalmente, esta situación brindaría la oportunidad de redefinir el emprendimiento en términos de la ESS y aportar valores propios de la ESS a la economía hegemónica. Sin embargo, en múltiples ocasiones vemos el proceso inverso: la economía hegemónica redefine los valores propios de la ESS para adaptarlos a las exigencias del mercado capitalista ${ }^{16}$.

\section{A modo de conclusión}

En general, el crecimiento y la expansión de la ESS ha permitido visibilizar y socializar algunas de las propuestas. Ahora bien, a lo largo de este crecimiento se han establecido relaciones de dependencia con las instituciones. Por ejemplo, a través de las subvenciones y el trabajo asalariado asociado a las mismas. En la actualidad, existe un debate abierto sobre el modelo de crecimiento y escalabilidad de la ESS popularizado como la "burbuja de la ESS". No obstante, se trata de un término metafórico que suscita la reflexión, pero con el que algunos cooperativistas no se sienten cómodos.

Por otro lado, también hay casos concretos en los que la expansión y crecimiento de la ESS ha supuesto cierta domesticación de las propuestas, en la medida que las líneas estratégicas han sido modificadas o ha habido una aceleración de los ritmos de trabajo que ha provocado cambios en el seno de las cooperativas. En general, la socialización, siempre relativa, transcurre en paralelo a procesos de apropiación e integración. Por un lado, las instituciones ven multiplicadas sus inversiones gracias a la motivación política, ideológica y vital de las personas involucradas en la ESS. Por otro lado, permiten mantener a las experiencias ciertos signos de identidad no amenazadores, siempre y cuando se sometan por completo a las exigencias hegemónicas básicas (Narotzky, 2004: 249). Este fenómeno en el que las empresas cooperativas se acercan a las empresas de la economía mercantil ha sido definido por Laville (2004) como isomorfismo institucional. Este autor plantea la necesidad de establecer una serie de reglas internas e institucionales que favorezcan la "resistencia a la mercantilización e institucionalización por los poderes instituidos" (Laville, 2004: 230).

Finalmente, el desarrollo de proyectos de auto-ocupación colectiva está de acuerdo con el desarrollo del emprendimiento en un panorama de desmantelamiento del Estado, su des-responsabilización y la transferencia de responsabilidades a los individuos para imaginar y construir sus propios puestos de trabajo. Esta transferencia de responsabilidades favorece la segmentación social y corre el riesgo de aumentar

16 Gran parte de las ponencias del último Global Social Economy Forum (GSEF) celebrado en Bilbao en octubre de 2018 es una muestra de la importancia de poner en el centro de los proyectos de economía social el valor de la competitividad con el fin de ser una opción viable en el mercado capitalista. Se apuesta por desarrollar un modelo sostenible con valores sociales pero que al mismo tiempo sea capaz de competir en la economía de mercado global: "La cooperación es imprescindible, pero hay que ir de la mano de la competitividad" (Txomin Garcia. Presidente de Laboral Kutxa y Vice-presidente de la Unión Nacional de Cooperativas de Crédito. Sesión de clausura del GSEF) 
las ya existentes desigualdades sociales. Además, estos puestos de trabajo tienen un especial interés porque son portadores de valores sociales añadidos que cumplen un papel crucial tras la desarticulación del estado del bienestar y los recortes sociales.

Por último, esta etnografía nos muestra las relaciones entre las estructuras socioeconómicas y políticas con las experiencias encarnadas de los y las cooperativistas y vincula las dimensiones macroeconómicas con las microeconómicas (y las macropolíticas con las micropolíticas) (Pérez-Orozco, 2012; Sarkis, 2018).

\section{Bibliografía}

Alquézar, Raquel (2015). La economía social y solidaria y las finanzas éticas: entre el valor social y el valor de mercado. Tesis doctoral. Facultad de Historia y Geografía. Universidad de Barcelona.

Alquézar, Raquel; Homs, Patricia; Morelló, Núria; et al. (2014). "Prácticas cooperativas: ¿estrategias de supervivencia, movimientos "alternativos" o reincrustación capitalista?". Ars \& Humanitas, 8(1): 151-166. doi: http://doi.org/10.4312/ars.8.1.151-166

Ben-ner, Avner (1988). "The life cycle of worker-owned firms in market economies". Journal of Economic Behavior and Organization, 10: 287-313. doi: https://doi.org/10.1016/01672681(88)90052-2.

Bouchard, Marie; Bourque, Gilles; Lévesque, Benoît (2000). "L'évaluation de l'économie sociale dans la perspective des nouvelles formes de régulation socio-économique de l'intérêt général". Cahiers du CRISES, Working Paper, nº 0013. doi: http://doi. org/10.7202/1002235ar.

Bourdieu, Pierre (1988 [1979]). La Distinción. Criterios y bases sociales del gusto. Madrid: Taurus.

Castell, Pere; Ferrer, Roser; Olivella, Marta; et al. (2016). Informe sobre la situació del treball autònom 2015. Consell de Treball, Econòmic i Social de Catalunya. Col-lecció Estudis i Informes, $n^{\circ} 41$.

Chayanov, Aleksandr Vasílievitx (1991 [1927]). The Theory of Peasant Co-operatives. Gran Bretaña: I.B.Tauris.

COM (2011). Iniciativa en favor del emprendimiento social. Construir un ecosistema para promover las empresas sociales en el centro de la economía y la innovación sociales. 682 final.

Coraggio, José Luis (2004). "Economía del Trabajo", en A. D. Cattani (Ed.), La otra economía. Buenos Aires: Editorial Altamira, 151-163.

EGSEC (Expert Groups on Social Entrepreneurship of the Commission) (2016). El futuro de la economía social y de las empresas que operan en este sector. Bélgica: European Union.

Elson, Diane (2001) "For an emancipatory socio-economics", paper para la conferencia UNRISD “The Need to Rethink Development", 7-8 septiembre, Ciudad del Cabo, Sudáfrica.

Evans, James (2011). "Resilience, ecology and adaptation in the experimental city". Transactions of the Institute of British Geographers, 36: 223-237 doi: https://doi. org/10.1111/j.1475-5661.2010.00420.x.

Federici, Silvia (2013). Revolución en punto cero: trabajo doméstico, reproducción y luchas feministas. Madrid: Traficantes de Sueños.

Fernández, Anna; Miró, Iván (2016). L'economia social i solidària a Barcelona. Barcelona: La Ciutat Invisible, SCCL. y Comisionado de Economia Cooperativa, Social y Solidària, Ayuntamiento de Barcelona. 
Friedmann, Harriet (1978). "World market, state, and family farm: social bases of household production in the era of wage labor". Comparative Studies in Society and History, 20 (4):545-86. doi: https://doi.org/10.1017/S001041750001255X.

Galt, Ryan E. (2013). “The Moral Economy Is a Double-edged Sword: Explaining Farmers' Earnings and Self-exploitation in Community-Supported Agriculture". Economic Geography, 89: 341-365 doi: https://doi.org/10.1111/ecge.12015.

Gibson-Graham, Julie Katherine (2006). A Postcapitalist Politics. Minnesota: University of Minnesota Press.

Gibson-Graham, Julie Katherine (2008). "Diverse Economies: Performative Practices for 'Other Worlds"'. Progress in Human Geography, no 32 (5): 613-632.

Homs, Patricia (2019). En prensa. "Resilient austerity and values. Social economy and entrepreneurship in Catalonia" en Narotzky, S. (ed.) Grassroots Economies: Living with Austerity in Southern Europe Londres: Pluto Press

Homs, Patricia; Narotzky, Susana (2019). "Within and beyond market allocation systems. The case of organic food cooperatives in Catalonia" en Sinischalchi, V.; Harper, K (eds) Food Values in Europe: Economies, Ideologies, and Power in Practice. Londres: Bloomsbury

Jacobs, Keith; Manzi, Tony (2013). "New Localism, Old Retrenchment: The "Big Society", Housing Policy and the Politics of Welfare Reform". Housing, Theory and Society, 30 (1): 29-45. doi: https://doi.org/10.1080/14036096.2012.683293.

Kasmir, Sharryn (2016). "The Mondragon Cooperatives and Global Capitalism: A Critical Analysis". New Labor Forum, 25(1): 52-59. doi: https:doi. org/10.1177/1095796015620424.

Lautier, Bruno (2003). "Les limites de l'économie informelle comme alternative à la mondialisation libérale". Revue du MAUSS, 21, 2003/1: 198-214. doi: https://doi. org/10.3917/rdm.021.0198.

Laville, Jean-Louis (2004). "El marco conceptual de la economía solidaria", en Laville, J-L. (ed.), Economía social y solidaria. Una visión europea. Buenos Aires: Altamira, 207-235.

Laville, Jean-Louis (2013). "The Social and Solidarity Economy. A Theoretical and Plural Framework". Draft paper prepared for the UNRISD Conference Potential and Limits of Social and Solidarity Economy 6-8 May 2013, Geneva, Switzerland.

MacKinnon, Danny; Driscoll, Kate (2012). "From resilience to resourcefulness: A critique of resilience policy and activism". Progress in Human Geography, 37(2) 253-270. doi: https://doi.org/10.1177/0309132512454775.

Mansilla, Elba; Grenzner, Joana; Alberich, Sílvia (2014). Femení plural. Les dones a l'economia cooperativa. Informe. Barcelona: Diputació de Barcelona.

Martín-Mayor, Adrià; Homs, Patricia; Flores-Pons, Gemma (2017). El canvi d'escala: un revulsiu per a la sostenibilitat del cooperativisme agroecologic? Informe. Barcelona: Fundació Roca i Galès y ACCID

Martínez, Laureno; Bogino Victoria (2015). "La instrumentación en España de las políticas europeas de emprendimento ¿creación de empleo o profundización de la crisis del empleo asalariado?". Revista Eletrônica de Ciência Política, 6 (1). doi: https://doi.org/10.5380/ recp.v6i1.37493.

Mateu, Paula (2016). "Valors que depassen el cooperativisme". Revista anual d'anàlisi $i$ reflexió sobre el món cooperatiu, 5: 46-53.

McCabe, Angus (2010). "Below the Radar in a Big Society? Reflections on community engagement, empowerment and social action in a changing policy context". Third Sector Research Centre, Working Paper 51. 
Moulaert, Frank; Alieni, Oana (2005). «Social Economy, Third Sector and Solidarity Relations: A Conceptual Synthesis from History to Present». Urban Studies, 42 (11): 2037-2053. doi: https://doi.org./10.1080/00420980500279794.

Narotzky, Susana (2004). Antropología económica. Nuevas tendencias. Santa Cruz de Tenerife: Melusina.

Narotzky, Susana; Besnier, Niko (2014). "Crisis, Value and Hope: Rethinking the Economy: An introduction to Suplement 9". Current Anthropology, 55 (59): 4-16. doi: https://doi. org/10.1086/676327.

Oppenheim, Carey; Cox, Ed; Platt, Reg (2010). Regeneration through co-operation: creating a framework for communities to act together. Manchester: Co-Operatives UK.

Pérez Baró, Albert (1989). Història de les cooperatives a Catalunya. Barcelona: Crítica.

Pérez-Orozco, Amaia (2012). "Crisis multidimensional y sostenibilidad de la vida". Investigaciones feministas, 1: 29-53. doi: http://dx.doi.org/10.5209/rev_INFE.2011. v2.38603.

Pérez-Orozco, Amaia (2014). Subversión feminista de la economía. Madrid: Traficantes de sueños.

Salvatori, Gianluca (2011). "Las empresas sociales y cooperativas dentro del nuevo paradigma: porque en Europa la realidad niega la ideología”. Euricse Working Papers, $\mathrm{n}^{\mathrm{o}} .21 \mid 11$.

Salvia, Agustín (2004). Crisis del empleo y nueva marginalidad: el papel de las economías de la pobreza en tiempos de cambio social. Jornadas Internacionales ICALA "Trabajo, riqueza e inclusión", Río Cuarto.

Sarkis, Diana (2018). “"Muerta a trabajar'. Consideraciones feministas sobre la crisis (de la reproducción social) en Vélez Málaga (España)". Revista Andaluza de Antropología, 14: 89-107.

Seminario de Economía Crítica Taifa (2006). "Del pleno empleo a la plena precariedad". Informes de economia TAIFA, $\mathrm{n}^{\mathrm{0} 3}$.

Serrano, Eloi; Crespo, Patricia; Celma, Dolors et al. (2017). Cooperatives i crisi econòmica ¿una relació causal? Evolució de les cooperatives catalanes creades des del 1970 fins el 2016. Informe. Cátedra de Economía Social. Fundació Roca i Galés y Accid.

Walker, Jeremy; Cooper, Melinda (2011). "Genealogies of Resilience From Systems Ecology to the Political Economy of Crisis Adaptation". Security Dialogue, 14(2): 143-160. doi: https://doi.org/ 10.1177/0967010611399616.

\section{Recursos electrónicos:}

La Ciutat Invisible [http://www.laciutatinvisible.coop/] Consulta: septiembre 2018 Barris i crisi [https://barrisicrisi.wordpress.com/] Consulta: septiembre 2018 